\title{
Reversal of Anomeric Selectivity with $O$-Glycosyl Trichloroacetimidates as Glycosyl Donors and Thiols as Acceptors Under Acid/Base Catalysis
}

\author{
Amit Kumar ${ }^{[a]}$ and Richard R. Schmidt ${ }^{*[a, b]}$
}

Keywords: Carbohydrates / Glycosylation / Thioglycosylation / Reaction mechanisms

Boron trifluoride or trimethylsilyl trifluoromethanesulfonate catalysed the generation of thioglycosides from O-glucopyranosyl or O-galactopyranosyl trichloroacetimidates and thiols giving mainly or exclusively a-thioglycosides. However, the same reactions with phenylboron difluoride as catalyst are highly $\beta$-selective. An $S_{\mathrm{N}} 2$-type reaction course under acid/base catalysis is invoked by these and previous results.

\section{Introduction}

Glycosidation reactions catalysed by boron trifluoride with $O$-glucopyranosyl trichloroacetimidate $1 \alpha$ as donor, ${ }^{[1,2]}$ alkanethiols as acceptors (HXR: $\mathrm{X}=\mathrm{S}$ ) and molecular sieves as drying agent in $\mathrm{CH}_{2} \mathrm{Cl}_{2}$ as solvent at $-42^{\circ} \mathrm{C}$ afforded $\alpha$-anomeric alkyl thioglycosides with retention of configuration (Scheme 1). ${ }^{[2]}$ Because alcohols (HXR: $X=$ O) exhibit under these acid catalysis reaction conditions much lower $\alpha$ selectivity, ${ }^{[3]}$ the generally accepted $\mathrm{S}_{\mathrm{N}}$ 1-type mechanism (Scheme 1 , path a) ${ }^{[4,5]}$ via $\mathrm{BF}_{3}$-donor adduct $\mathbf{I}$, the generation of glycosyl cation intermediate II and the subsequent more or less diastereocontrolled reaction with the acceptor to yield $1 \mathrm{Z \alpha}$ and $1 \mathrm{Z \beta}$ was questioned for the quite nucleophilic alkanethiols. Instead, an intramolecular reaction course via an initial attack of the thiol group at the activated iminium carbon leading to adduct III and then rearrangement of the adduct to the $\alpha$-product $1 \mathrm{Za}$. was discussed (Scheme 1, path b). ${ }^{[2]}$

Recently it was found that phenylboron difluoride $\left(\mathrm{PhBF}_{2}\right)$ is unable to activate glycosyl donor $1 \alpha$. However, the $\mathrm{PhBF}_{2}$.alcohol adduct leads, with inversion of configuration, readily to glycosides with high $\beta$ selectivity, particularly when the inverse procedure (IP) was applied (i.e., addition of the donor to a solution of the acceptor and catalyst). ${ }^{[6]}$ For these highly stereocontrolled reactions a hydrogen-bond-mediated acid/base-catalysed intramolecular $\mathrm{S}_{\mathrm{N}}$ 2-type reaction course was proposed (Scheme 1, path $\mathrm{c}$, $\mathrm{X}=\mathrm{O}$ ) that is also supported by other results. ${ }^{[6,7]}$ This reaction course is closely related to the reaction mechanism

[a] Fachbereich Chemie, Universität Konstanz, Fach 725, 78457 Konstanz, Germany Fax: $+49-7531-883135$

E-mail: richard.schmidt@uni-konstanz.de

[b] Chemistry Department, Faculty of Science, King Abdulaziz University,

Jeddah 21589 , Saudi Arabia found for glycosyltransferase-catalysed glycosidation reactions. ${ }^{[8]}$ As boron has a lower affinity to the sulfur of thiols than to the oxygen of alcohols, ${ }^{[9]}$ it was of interest to determine whether $\mathrm{PhBF}_{2}$ will catalyse the reactions with thiols at all and when thioglycosides are obtained if prior $\mathrm{PhBF}_{2}$ thiol adduct formation $(I V ; X=S)$ will lead under acid/base catalysis via $S_{N}$ 2-type transition state $V$ to a reversal of the anomeric selectivity and to $\beta$-thioglycoside 1ZP. Thus, further strong support for this reaction course would be obtained.

\section{Results and Discussion}

The main focus of this work was on the use of $\mathrm{PhBF}_{2}$ as catalyst; for comparison, some studies with $\mathrm{BF}_{3} \cdot \mathrm{OEt}_{2}$ and trimethylsilyl trifluoromethanesulfonate (TMSOTf) as catalysts are reported (Table 1). As previously found, the reaction of glucosyl donor $1 \alpha$ with isopropanethiol (A) as acceptor (Figure 1) in the presence of molecular sieves (4 $\AA$ ) and $\mathrm{BF}_{3} \cdot \mathrm{OEt}_{2}$ as catalyst afforded at $-42{ }^{\circ} \mathrm{C}$ under the normal procedure (NP: addition of the catalyst to a solution of the donor and acceptor) the $\alpha$ anomer 1 A $\alpha$ (entry 1 ). ${ }^{[2.10]}$ When this reaction was carried out under IP conditions at $-78^{\circ} \mathrm{C}$ without the addition of molecular sieves, a mixture of anomers with a slight preference for $\mathbf{1 A \beta}$ was obtained (entry 2). With the widely employed TMSOTf as catalyst under NP and IP conditions, practically the same results were obtained with a preference for the $\alpha$ anomer (entries 3 and 4) and with $\mathrm{B}\left(\mathrm{SC}_{3} \mathrm{H}_{7}\right)_{3}$ as acceptor and TMSOTf as catalyst a very slow reaction was observed under these conditions (entry 5). Hence, the result with $\mathrm{PhBF}_{2}$ as catalyst was of great interest as this reagent is too weak to activate donor $\mathbf{1} \alpha$ in the absence of an acceptor. Only the adduct IV (Scheme 1, $\mathrm{X}=\mathrm{S}$ ) will react and indeed ${ }^{19} \mathrm{~F}$ NMR analysis indicated an interaction between $\mathrm{PhBF}_{2}$ and the thiol groups (no change was observed in the ${ }^{1} \mathrm{H}$ NMR spectrum). The reaction of $1 \alpha$ with $A$ under NP conditions led mainly 


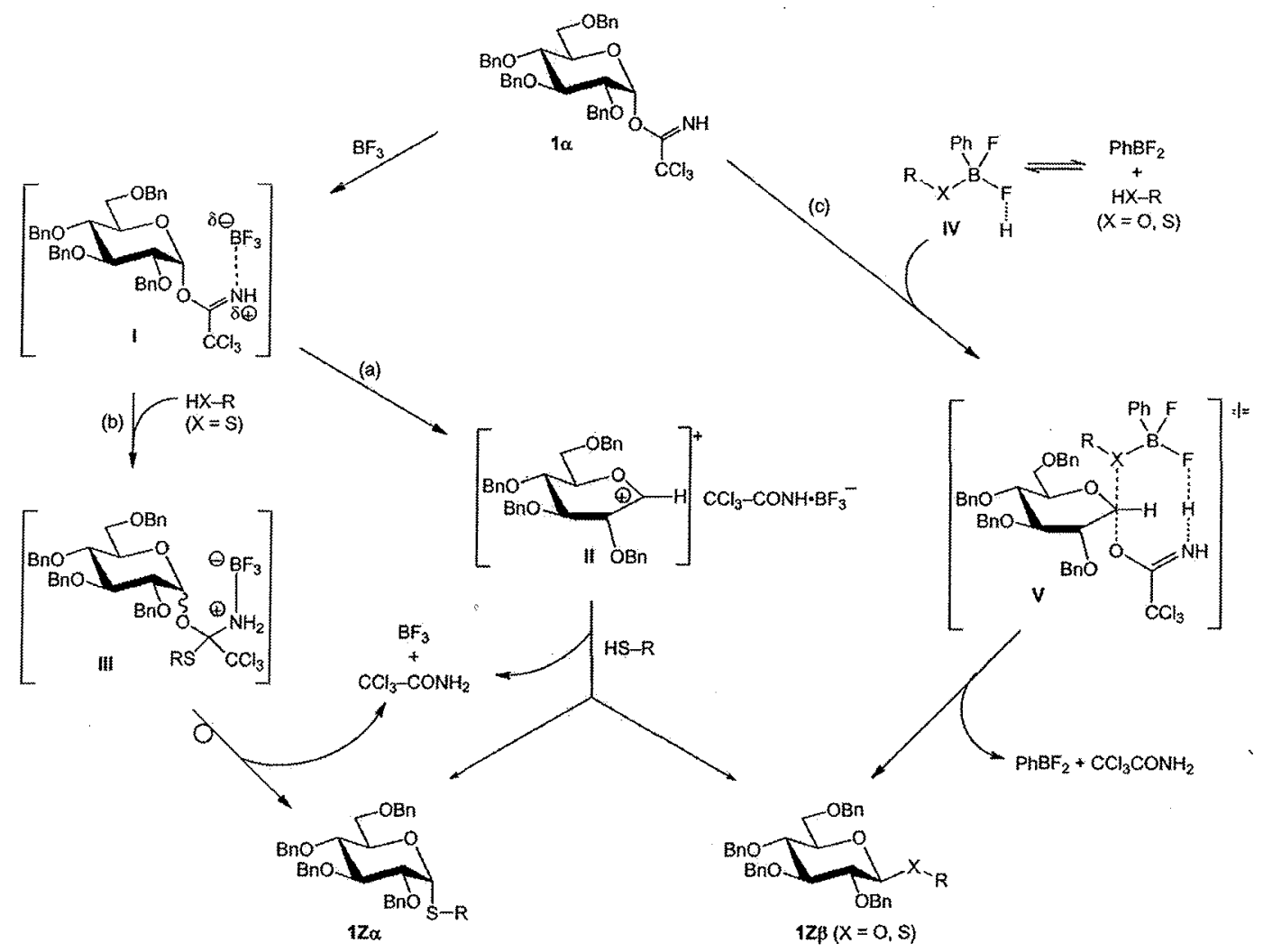

Scheme 1. Activation of $O$-glycosyl trichloroacetimidates with boron fluorides in the presence of thiols and alcohols as acceptors.

Table 1. Reactions of $1 \alpha-4 \alpha$ with different thiols as acceptors and different glycosidation catalysts in $\mathrm{CH}_{2} \mathrm{Cl}_{2}$ as solvent.

\begin{tabular}{|c|c|c|c|c|c|c|c|c|}
\hline Entry & Donor & Acceptor & $\begin{array}{c}\text { Cat. } \\
\text { (0.1 equiv. })\end{array}$ & \multicolumn{3}{|c|}{ Reaction conditions } & $\begin{array}{l}\text { Product }{ }^{\mid b]} \\
\text { (\% yield })\end{array}$ & $\beta / \mu^{[c]}$ \\
\hline 1 & $1 \alpha$ & $\mathbf{A}$ & $\mathrm{BF}_{3} \cdot \mathrm{OEt}_{2}$ & n.p. & -42 & 1 & 1A $(85 \%)$ & $u^{[d]}$ \\
\hline 2 & $1 \alpha$ & A & $\mathrm{BF}_{3} \cdot \mathrm{OEt}_{2}$ & i.p. & -78 & 0.5 & $1 \mathbf{A}(88 \%)$ & $1.5: 1$ \\
\hline 3 & $1 \alpha$ & A & TMSOTf & n.p. & -78 & 0.2 & $1 \mathrm{~A}(81 \%)$ & $1: 2.4$ \\
\hline 4 & $1 \alpha$ & A & TMSOTf & i.p. & -78 & 0.2 & $1 \mathrm{~A}(80 \%)$ & $1: 2.4$ \\
\hline $5^{[e]}$ & $1 \alpha$ & $\mathrm{B}\left(\mathrm{SC}_{3} \mathrm{H}_{7}\right)_{3}$ & TMSOTf & n.p. & -78 & 0.7 & slow reaction & - \\
\hline 6 & $1 \alpha$ & $\mathbf{A}$ & $\mathrm{PhBF}_{2}$ & n.p. & -78 & 1 & 1A $(84 \%)$ & $5: 1$ \\
\hline 7 & $1 \alpha$ & $\mathbf{A}$ & $\mathrm{PhBF}_{2}$ & i.p. & -78 & 1 & $\mathbf{1 A}(79 \%)$ & $8: 1$ \\
\hline 8 & $1 \alpha$ & $\mathbf{A}$ & $2-\mathrm{PhC}_{6} \mathrm{H}_{4} \mathrm{BF}_{2}$ & i.p. & -78 & 0.7 & $1 \mathrm{~A}(83 \%)$ & $8: 1$ \\
\hline 9 & $1 u$ & B & $\mathrm{PhBF}_{2}$ & i.p. & -78 & 1 & $1 B(81 \%)$ & $7: 1$ \\
\hline 10 & $1 \alpha$ & $\mathrm{C}$ & $\mathrm{PhBF}_{2}$ & i.p. & -78 & 1.2 & $1 \mathrm{C}(76 \%)$ & $15: 1$ \\
\hline 11 & $1 \alpha$ & D & $\mathrm{PhBF}_{2}$ & i.p. & -78 & 1 & iD $(78 \%)$ & $10: 1$ \\
\hline 12 & $1 \alpha$ & $\mathbf{E}$ & $\mathrm{PhBF}_{2}$ & i.p. & -78 & 2 & 1E $(65 \%)$ & $4: 1$ \\
\hline 13 & $2 \alpha$ & A & $\mathrm{PhBF}_{2}$ & i.p. & -78 & 1 & $2 \mathrm{~A}(73 \%)$ & $15: 1$ \\
\hline 14 & $2 \alpha$ & B & $\mathrm{PhBF}_{2}$ & i.p. & -78 & 0.7 & $2 B(81 \%)$ & $16: 1$ \\
\hline 15 & $2 a$ & C & $\mathrm{PhBF}_{2}^{-}$ & i.p. & -78 & 1 & $2 \mathrm{C}(76 \%)$ & $16: 1$ \\
\hline 16 & $3 a$ & $A$ & $\mathrm{PhBF}_{2}$ & i.p. & -78 & 2 & $\mathbf{3 A}(77 \%)$ & $15: 1$ \\
\hline 17 & 30 & B & $\mathrm{PhBF}_{2}$ & i.p. & -78 & 1.5 & 3B $(78 \%)$ & $16: 1$ \\
\hline 18 & $3 \alpha$ & $\mathrm{C}$ & $\mathrm{PhBF}_{2}$ & i.p. & -78 & 2 & $3 \mathrm{C}(71 \%)$ & $16: 1$ \\
\hline 19 & $4 \alpha$ & $\mathbf{A}$ & $\mathrm{PhBF}_{2}$ & i.p. & -78 & 1.2 & $4 \mathrm{~A}(72 \%)$ & $25: 1$ \\
\hline 20 & $4 \alpha$ & B & $\mathrm{PhBF}_{2}$ & i.p. & -78 & 1 & $4 \mathrm{~B}(70 \%)$ & $15: 1$ \\
\hline 21 & $4 t$ & $\mathrm{C}$ & $\mathrm{PhBF}_{2}$ & i.p. & -78 & 1.2 & $4 \mathrm{C}(76 \%)$ & $16: 1$ \\
\hline 22 & $4 a$ & $\mathbf{E}$ & $\mathrm{PhBF}_{2}$ & i.p. & -78 & 2 & $4 \mathrm{E}(67 \%)$ & $10: 1$ \\
\hline
\end{tabular}

[a] n.p.: normal procedure; i.p.: inverse procedure; for an explanation, see text. [b] Isolated yields. [c] The $\alpha / \beta$ ratio was determined by ${ }^{1} \mathrm{H}$ NMR signal integration. [d] Reported in ref. ${ }^{[1]}$. [e] With 1 equiv. of TMSOTf as promoter a slow reaction to an anomeric mixture of 1B took place $(2 \mathrm{~h}$, yield $55 \%, \beta / \alpha \approx 1: 2)$. 


\section{Acceptors}

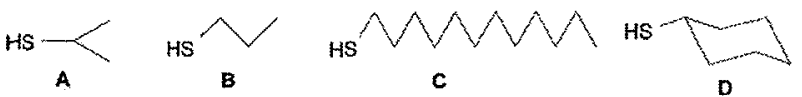

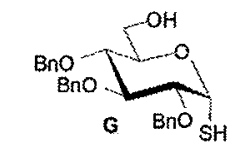

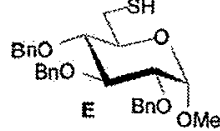<smiles>OCCCCS</smiles>

\section{Donors}
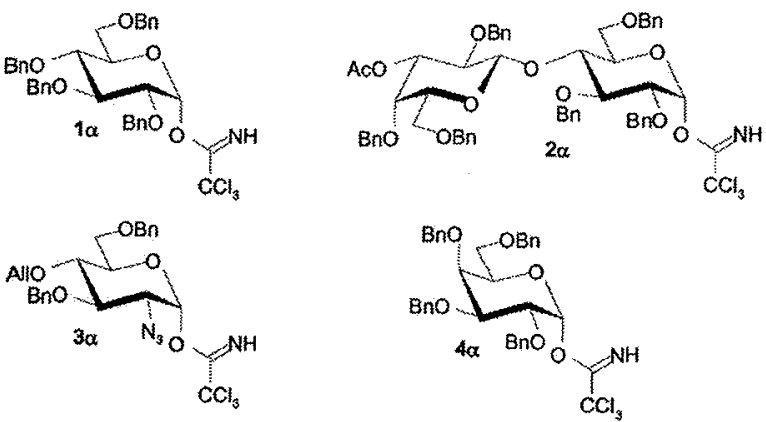

Figure 1. Glycosyl donors and acceptors investigated in this study.

to $\beta$ anomer $1 \mathrm{AB}$ (entry 6 ). However, as expected, IP conditions enhanced the formation of $\mathbf{1 A \beta}$ (entry 7 ), thereby supporting the proposed $S_{\mathrm{N}}$ 2-type transition state $\mathbf{V}$ (Scheme 1). Therefore these reaction conditions were employed in the following studies. The introduction of an $o$ phenyl group into the phenylboron moiety (entry 8) had only a minor effect on the result although an influence on the conformational preferences in the transition state was expected. Other acceptors, such as propanethiol (B), do-

decanethiol (C), cyclohexanethiol (D) and methyl 2,3,4-triO-benzyl-6-deoxy-6-mercapto- $\alpha$-D-glucopyranoside (E), ${ }^{[1]}$ showed similar strong preferences for the $\beta$ anomers $\mathbf{1 B} \beta,^{\left[{ }^{[2]}\right.}$ $1 \mathrm{C} \beta,^{[13]} 1 \mathrm{D} \beta^{[14]}$ and $1 \mathrm{E} \beta$, respectively (entries 9-12). Also, other glycosyl donors, such as lactosyl trichloroacetimidate $2 \alpha,{ }^{[6]}$ 2-azido- $\alpha$-1)-glucopyranosyl trichloroacetimidate $3 \alpha^{[15]}$ and $\alpha$-D-galactosyl trichloroacetimidate $4 \alpha,{ }^{[16]}$ gave under the standard reaction conditions mainly $\beta$ products $\left(2 \mathrm{~A} \beta-2 \mathrm{C} \beta, 3 \mathrm{~A} \beta-3 \mathrm{C} \beta, 4 \mathrm{~A} \beta, 4 \mathrm{~B} \beta{ }^{[10]} 4 \mathrm{C} \beta^{[17]}\right.$ and $4 \mathrm{E} \beta$; entries 13-22). Hence, the envisaged reversal of anomeric selectivity in comparison with the more common glycosidation procedures (entries 1 and 3) was confirmed, thus strongly supporting the initial formation of an adduct between $\mathrm{PhBF}_{2}$ and the thiol group and subsequent $\mathrm{S}_{\mathrm{N}} 2$-type reaction with the glycosyl donor.

Because boron has a higher affinity to hydroxy groups than thiols, ${ }^{[9]}$ comparison studies were of interest. To this end, 4-mercaptobutanol (F) was selected as the acceptor and under the standard conditions it was treated with donors $1 \alpha-3 \alpha$ (Scheme 2). As expected, only $\beta$-selective reactions at the hydroxy group of $\mathbf{F}$ were observed leading essentially to $1 F \beta-3 F \beta$, respectively; these compounds were characterized as their $S$-acetyl derivatives $1 \mathrm{Fa} \beta-3 \mathrm{Fa} \beta$. The observed chemoselectivity offers various possibilities for targeted functional group variations without resorting to protecting group manipulations. Therefore, as a more interesting case, 2,3,4-tri- $O$-benzyl- $\alpha$-1)-glucopyranosylthiol $(\mathbf{G})^{[18]}$ was also investigated as acceptor with $1 \alpha$ as donor. With $\mathrm{PhBF}_{2}$ as catalyst under the standard reaction conditions in a highly $\beta$-selective reaction exclusive hydroxy group attack was observed leading mainly to $1 G \beta{ }^{[18]}$ Thus, the strong interaction between boron and oxygen outbalancing the greater nucleophilicity of the sulfur is confirmed.
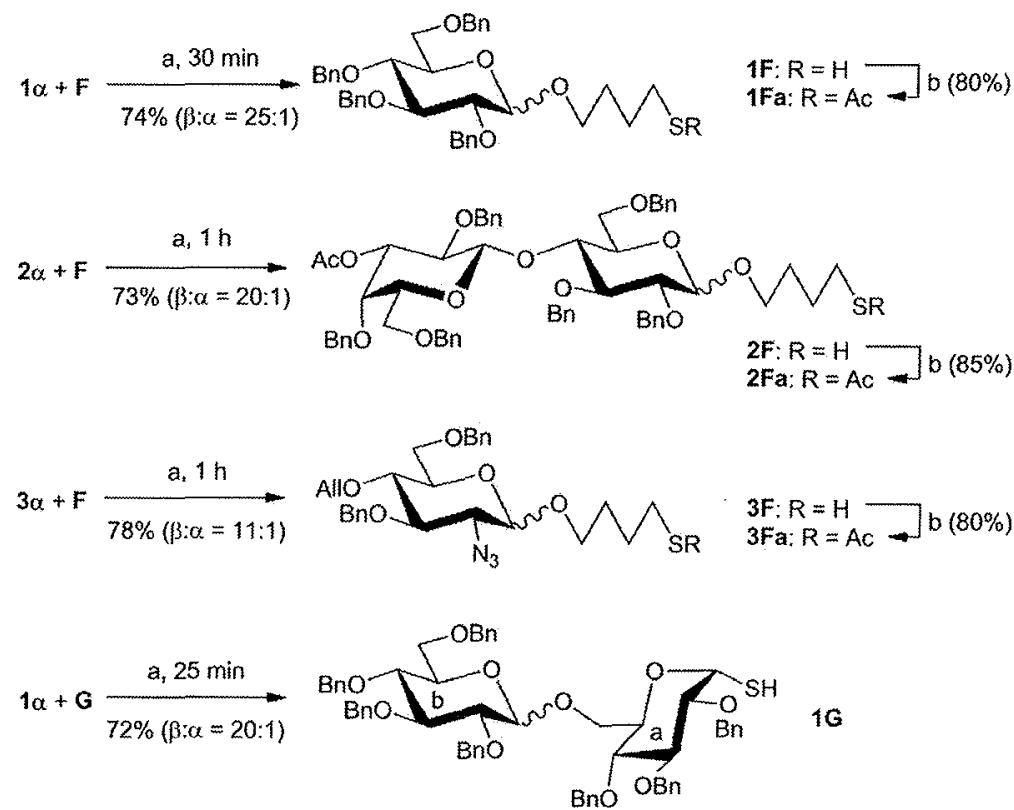

Scheme 2. Reactions with mercapto-containing alcohols. Reagents and conditions: (a) i.p.: $\mathrm{PhBF}_{2}, \mathrm{CH}_{2} \mathrm{Cl}_{2},-78^{\circ} \mathrm{C} ;$ (b) $\mathrm{Ac} \mathrm{c}_{2} \mathrm{O}, \mathrm{Pyr}, \mathrm{DMAP}$, r.t. 


\section{Conclusions}

O-Glycosyl trichloroacetimidates as donors and thiols as acceptors gave under standard acid catalysis conditions preferentially or exclusively $\alpha$-thioglycosides whereas acid/ base catalysis with $\mathrm{PhBF}_{2}$ led mainly to $\beta$-thioglycosides. As the glycosyl donors employed do not provide anchimeric assistance the $\beta$ selectivity strongly supports the previously invoked hydrogen-bond-mediated acid/base-catalysed intramolecular $\mathrm{S}_{\mathrm{N}} 2$-type reaction course. Acceptors with unprotected thiol and hydroxy groups exhibit a high affinity for boron through the hydroxy groups as only $\beta$-selective glycosidation reactions with the hydroxy moieties were observed with $\mathrm{PhBF}_{2}$ as catalyst.

\section{Experimental Section}

General Methods: Solvents were purified by standard procedures ${ }^{1} \mathrm{H}$ and ${ }^{13} \mathrm{C}$ NMR spectra were recorded at $22{ }^{\circ} \mathrm{C}$ with a Bruker spectrometer ( ${ }^{1} \mathrm{H}: 400 \mathrm{MHz} ;{ }^{13} \mathrm{C}: 100 \mathrm{MHz}$ ). Tetramethylsilane (TMS) or the resonance of the undeuteriated solvent were used as internal standards (solvent $\mathrm{CDCl}_{3}:{ }^{1} \mathrm{H}: \delta=7.24 \mathrm{ppm} ;{ }^{13} \mathrm{C}: \delta=$ 77.25). Mass spectra were recorded with a Bruker ESI MS mass spectrometer. Thin-layer chromatography was performed on Merck silica gel $\left(60 \mathrm{~F}_{254}\right)$ plastic plates. Compounds were visualized by treatment with a solution of $\left(\mathrm{NH}_{4}\right)_{6} \mathrm{Mo}_{7} \mathrm{O}_{24} \cdot 4 \mathrm{H}_{2} \mathrm{O}(20 \mathrm{~g})$ and $\mathrm{Ce}\left(\mathrm{SO}_{4}\right)_{2}(0.4 \mathrm{~g})$ in sulfuric acid $(10 \%, 400 \mathrm{~mL})$ and then heating to $120^{\circ} \mathrm{C}$. Flash chromatography was performed on MN Silica gel $60(230-400 \mathrm{mesh})$ at a pressure of 0.2 bar. Optical rotations were measured at $22^{\circ} \mathrm{C}$ with a Büchi Polar-Monitor using the sodium $D$ line. Some of the thiols were used as commercial grade. Starting materials were obtained by following literature procedures. All reactions were performed in oven-dried glassware under dry nitrogen.

\section{Representative Procedures for the Thioglycosylation Reactions}

Procedure A - Inverse Procedure: The catalyst $\left[\mathrm{PhBF}_{2}\right.$, TMSOTf or $\mathrm{BF}_{3} \cdot \mathrm{OEt}_{2}$ (0.1 equiv.) in $\mathrm{CH}_{2} \mathrm{Cl}_{2}$ ] was added to a solution of the acceptor (1 equiv.) in $\mathrm{CH}_{2} \mathrm{Cl}_{2}$ at room temperature. The reaction mixture was then cooled to $-78^{\circ} \mathrm{C}$. The donor (1 equiv.) was dissolved in a minimum amount of $\mathrm{CH}_{2} \mathrm{Cl}_{2}$ and after cooling to $-78^{\circ} \mathrm{C}$ it was added to the reaction mixture at once. The mixture was stirred at the same temperature until TLC indicated complete consumption of the starting material. The reaction was quenched with aqueous $\mathrm{NaHCO}_{3}$ and extracted with $\mathrm{CH}_{2} \mathrm{Cl}_{2}$. The organic layer was washed with water, dried with $\mathrm{MgSO}_{4}$ and concentrated in vacuo. The crude product was purified by flash column chromatography with petroleum ether/EtOAc as eluent to afford the desired glycoside. This way in most cases only the major anomer was obtained (see data of the compounds).

Procedure B - Normal Procedure: The catalyst $\left[\mathrm{PhBF}_{2}\right.$ or TMSOTf (0.1 equiv.) in $\mathrm{CH}_{2} \mathrm{Cl}_{2}$ ] was added dropwise to a cooled solution $\left(-78^{\circ} \mathrm{C}\right)$ of the donor (1 equiv.) and acceptor (1 equiv.) in $\mathrm{CH}_{2} \mathrm{Cl}_{2}$. The mixture was stirred at the same temperature until TLC indicated complete consumption of the starting material. The reaction was then quenched with aqueous $\mathrm{NaHCO}_{3}$ and extracted with $\mathrm{CH}_{2} \mathrm{Cl}_{2}$. The organic layer was washed with water, dried with $\mathrm{MgSO}_{4}$ and concentrated in vacuo. The crude product was purified by flash column chromatography with petroleum ether/EtOAc as eluent to afford the desired glycosides.

Procedure C - Representative Procedure for the Acetylation: Acetic anhydride $(0.5 \mathrm{~mL})$, dry pyridine $(0.5 \mathrm{~mL})$ and a few crystals of
DMAP were added to a stirred solution of the hydroxy compound in dry $\mathrm{CH}_{2} \mathrm{Cl}_{2}(2 \mathrm{~mL})$ at room temperature. The reaction mixture was stirred at room temperature until TLC indicated complete consumption of the starting material $(12 \mathrm{~h})$. Then the reaction mixture was diluted with $\mathrm{CH}_{2} \mathrm{Cl}_{2}(10 \mathrm{~mL})$ and washed with diluted $\mathrm{HCl}$. The organic layer was dried with $\mathrm{MgSO}_{4}$ and concentrated in vacuo. The residue was purified by flash column chromatography with petroleum ether/EtOAc as elnent.

4-Mercaptobutyl 2,3,4,6-Tetra- $O$-benzyl- $\beta$-D-glucopyranoside (1F): The general procedure A for glycosylation afforded $1 F$ as a viscous oil; yield 74\%; $0 . / 3$ l:25. $R_{\mathrm{f}}=0.5$ (petroleum ether/EtOAc, 7:3). $[a]_{D}=+40.5\left(c=1.1, \mathrm{CHCl}_{3}\right)$. 'H NMR $\left(400 \mathrm{MHz}, \mathrm{CDCl}_{3}\right): \delta=$ 7.47-7.25 (m, $18 \mathrm{H}$, Ar-H), 7.21-7.11 (m, $2 \mathrm{H}, \mathrm{Ar}-\mathrm{H}), 4.95$ (dd, $J$ $=11.0,4.7 \mathrm{~Hz}, 2 \mathrm{H}, \mathrm{PhCH}), 4.80\left(\right.$ br. $\mathrm{t}, J_{\mathrm{app}}=10.4 \mathrm{~Hz}, 2 \mathrm{H}$, $\mathrm{PhCH}), 4.72(\mathrm{~d}, J=11.0 \mathrm{~Hz}, 1 \mathrm{H}, \mathrm{PhCH}), 4.62-4.56(\mathrm{~m}, 2 \mathrm{H}$, $\mathrm{PhCH}), 4.53(\mathrm{~d}, J=11.0 \mathrm{~Hz}, 1 \mathrm{H}, \mathrm{PhCH}), 4.41(\mathrm{~d}, J=7.8 \mathrm{~Hz}, 1$ $\mathrm{H}, 1-\mathrm{H}), 4.03-3.96\left(\mathrm{~m}, 1 \mathrm{H}, 1^{\prime}-\mathrm{H}\right), 3.73(\mathrm{dd}, J=9.0,5.3 \mathrm{~Hz}, 1 \mathrm{H}$, $6 \mathrm{a}-\mathrm{H}), 3.69-3.64(\mathrm{~m}, 2 \mathrm{H}, 5-\mathrm{H}, 6 \mathrm{e}-\mathrm{H}), 3.61$ (br. d, $J_{\text {ари }}=8.4 \mathrm{~Hz}, 1$ $\mathrm{H}, 3-\mathrm{H}), 3.56-3.54\left(\mathrm{~m}, 1 \mathrm{H}, 1^{\prime}-\mathrm{H}\right), 3.47-3.44(\mathrm{~m}, 2 \mathrm{H}, 2-\mathrm{H}, 4-\mathrm{H})$, $2.57(\mathrm{q}, J=6.8 \mathrm{~Hz}, 2 \mathrm{H}), 1.77-1.71(\mathrm{~m}, 4 \mathrm{H}), 1.33(\mathrm{t}, J=8.0 \mathrm{~Hz}$, $1 \mathrm{H}, \mathrm{SH}) \mathrm{ppm} .{ }^{13} \mathrm{C} \mathrm{NMR}\left(100 \mathrm{MHz}, \mathrm{CDCl}_{3}\right): \delta=138.8,138.7$, $138.4,138.3,128.6,128.5,128.2-127.4(\mathrm{~m}), 103.6(\mathrm{C}-1), 84.7,82.5$, $78.1,76.6,75.6,75.9,75.2,75.1,75.0,73.7,69.5,69.2,30.8,28.7$, 24.6 ppm. HRMS: calcd. for $\mathrm{C}_{38} \mathrm{H}_{44} \mathrm{NaO}_{6} \mathrm{~S}$ [M $\left.+\mathrm{Na}\right]^{+} 651.2751$; found 651.2773 .

4-Acetylthiobutyl 2,3,4,6-Tetra- $O$-benzyl-p-D-glucopyranoside (1Fa): Acetylation under standard conditions (Procedure C) afforded $1 \mathrm{Fa}$ as a white solid; yield $80 \% . R_{\mathrm{f}}=0.4$ (petroleum ether/EtOAc, 7:3). $[\alpha]_{\mathrm{D}}=+23.8\left(c=1.1, \mathrm{CHCl}_{3}\right) \cdot{ }^{\mathrm{t}} \mathrm{H}$ NMR $\left(400 \mathrm{MHz}, \mathrm{CDCl}_{3}\right): \delta=$ 7.45-7.23 (m, $18 \mathrm{H}, \mathrm{Ar}-\mathrm{H}), 7.23-7.12(\mathrm{~m}, 2 \mathrm{H}, \mathrm{Ar}-\mathrm{H}), 4.94$ (app dd, $\left.J_{\text {app }}=11.0,2.1 \mathrm{~Hz}, 2 \mathrm{H}, \mathrm{PhCH}\right), 4.83\left(\operatorname{app~t}, J_{\mathrm{app}}=11.0 \mathrm{~Hz}\right.$, $2 \mathrm{H}, \mathrm{PhC} H), 4.74(\mathrm{~d}, J=11.1 \mathrm{~Hz}, 1 \mathrm{H}, \mathrm{PhCH}), 4.62(\mathrm{~d}, J=$ $12.2 \mathrm{~Hz}, 1 \mathrm{H}, \mathrm{PhCH}), 4.55(\mathrm{dd}, J=11.5,7.8 \mathrm{~Hz}, 2 \mathrm{H}, \mathrm{PhC} H), 4.40$ $(\mathrm{d}, J=7.8 \mathrm{~Hz}, 1 \mathrm{H}, 1-\mathrm{H}), 4.03-3.91\left(\mathrm{~m}, 1 \mathrm{H}, 1^{\prime}-\mathrm{H}\right), 3.75(\mathrm{dd}, J=$ $10.8,2.0 \mathrm{~Hz}, 1 \mathrm{H}, 6 \mathrm{a}-\mathrm{H}), 3.71-3.66(\mathrm{~m}, 1 \mathrm{H}, 6 \mathrm{e}-\mathrm{H}), 3.66-3.61(\mathrm{~m}$, $1 \mathrm{H}, 5-\mathrm{H}), 3.56-3.53\left(\mathrm{~m}, 2 \mathrm{H}, \mathrm{I}^{\prime}-\mathrm{H}, 3-\mathrm{H}\right), 3.50-3.40(\mathrm{~m}, 2 \mathrm{H}, 2-\mathrm{H}$, 4-H), 2.98-2.89 (m, $2 \mathrm{H}), 2.33\left(\mathrm{~s}, 3 \mathrm{H}, \mathrm{COCH}_{3}\right), 1.78-1.64(\mathrm{~m}, 4$ H) ppm. ${ }^{13} \mathrm{C}$ NMR $\left(100 \mathrm{MHz}, \mathrm{CDCl}_{3}\right): \delta=195.8,138.7,138.5$, $138.2,138.1,128.3,128.2-127.4$ (m), $103.6(\mathrm{C}-1), 84.7,82.2,77.9$, $75.6,75.0,74.88,74.85,73.5,69.2,68.9,30.6,28.88,28.86$, 26.3 ppm. HRMS: calcd. for $\mathrm{C}_{40} \mathrm{H}_{46} \mathrm{NaO}_{7} \mathrm{~S}[\mathrm{M}+\mathrm{Na}]^{+}$693.2856; found 693.2871 .

Supporting Information (see footnote on the first page of this article): Synthetic methods and physical data for new compounds and ${ }^{1} \mathrm{H}$ and ${ }^{13} \mathrm{C}$ NMR spectra of all synthesized compounds.

\section{Acknowledgments}

This work was supported by the University of Konstanz and the Fonds der Chemischen Industrie.

[1] R. R. Schmidt, J. Michel, Angew. Chem. 1980, 92, 763-764; Angew. Chem. Int. Ed. Engl. 1980, 19, 731-732.

[2] R. R. Schmidt, M. Stumpp, Liebigs Ann. Chem. 1983, 12491256

[3] a) R. R. Schmidt, Angew. Chem. 1986, 98, 213-236; Angew. Chem. Int. Ed. Engl. 1986, 25, 212-235; b) R. R. Schmidt, W. Kinzy, Adv. Carbohydr. Chem. Biochem. 1994, 50, 21-123.

[4] A. V. Demchenko, Handbook of Chemical Glycosylation, WileyVCH, Weinheim, 2008.

[5] X. Zhu, R. R. Schmidt, Angew. Chem. 2009, 12l, 1932-1967; Angew. Chem. Int. Ed. 2009, 48, 1900-1934. 
[6] A. Kumar, V. Kumar, R. T. Dere, R. R. Schmidt, Org. Lett. 2011, 13, 3612-3615.

[7] A. Kumar, Y. Geng, R. R. Schmidt, Adv. Synth. Cat., accepted; DOI: $10.1002 / \mathrm{adsc} .201100933$.

[8] a) F. V. Rao, J. R. Rich, B. Rakić, S. Buddai, M. F. Schwartz, K. Johnson, C. Bowe, W. W. Wakarchuk, S. DeFrees, S. G. Withers, N. C. J. Strynadka, Nature Struct. Mol. Biol. 2009, 16, 1186-1188; b) J. I. Rearick, J. E: Sadler, J. C. Paulson, R. L. Hill, J. Biol. Chem. 1979, 254, 4444-4451; c) see also: K.-H. Jung, M. Müller, R. R. Schmidt, Chem. Rev 2000, 100, 44234442 .

[9] D. J. Grant, D. A. Dixon, J. Phys. Chem. A 2009, 113, 777787.

[10] Y. Du, M. Zhang, F. Yang, G. Gu, J. Chem. Soc. Perkin Trans. I 2001, 3122-3127.

[11] S.-S. Weng, Y.-D. Liu, C.-T. Chen, Org. Lett. 2006, 8, 56335636.
[12] F. K. Griffin, D. E. Paterson, P. V. Murphy, R. J. K. Taylor, Eur. J. Org. Chem. 2002, 1305-1322.

[13] H. Matsui, J.-1. Furukawa, T. Awano, N. Nishi, N. Sakairi, Chem. Lett. 2000, 326-327.

[14] T. Sato, Y. Fujita, J. Otera, H. Nozaki, Tetrahedron Lett. 1992 , $33,239-242$

[15] Y. Du, J. Liu, R. J. Linhardt, J. Carbohydr. Chem. 1997, 16, $1327-1344$.

[16] B. Wegmann, R. R. Schmidt, J. Carbohydr: Chem. 1987, 6, 357375.

[17] S.-H. Son, C. Tano, T. Furnike, N. Sakairi, Tetrahedron Lett. 2008, 49, 5289-5292.

[18] R. T. Dere, A. Kumar, V. Kumar, X. Zhu, R. R. Schmidt, $J$ Org. Chem. 2011, 76, 7539-7545. 